\title{
EDITORIAL
}

\section{The role of monocytes-macrophages in vasculogenesis in multiple myeloma}

\section{Leukemia (2009) 23, 1535-1536; doi:10.1038/leu.2009.55}

Inflammatory cells regulate endothelial cell functions related to physiological angiogenesis as well as inflammatory and tumorassociated angiogenesis. It was Rudolf Virchow in 1863, who critically recognized the presence of inflammatory cells infiltrating neoplastic tissues, and first established a causative connection between the 'lymphoreticular infiltrate' at sites of chronic inflammation and cancer. In neoplastic tissues, inflammatory cells act in concert with tumor cells, stromal cells and endothelial cells to create a microenvironment that is critical for the survival, development and diffusion of the neoplastic mass. These synergies may represent important mechanisms for tumor development and metastasis by providing an efficient vascular supply and an easy escape pathway.

Cells belonging to the monocyte-macrophage lineage are a major component of the leukocyte infiltration in tumors and there is growing evidence that they are part of inflammatory circuits that promote tumor progression, and favor invasion and metastasis. ${ }^{1,2}$ The stimulating effect exerted by tumor-associated macrophages on the growth of the tumor mass is partly related to the angiogenic potential of these cells. Tumor-associated macrophages are a rich source of potent proangiogenic cytokines and growth factors, such as vascular endothelial growth factor, tumor necrosis factor alpha, interleukin-8 and fibroblast growth factor-2. They also express a broad array of angiogenesis-modulating enzymes, including matrix metalloproteinases (MMPs)-2, -7, -9, and -12 (MMP-2, MMP-7, MMP-9 and MMP-12, respectively), cycloxygenase-2 and colony-stimulating factor- $1 . .^{3-7}$ The many proangiogenic factors secreted by macrophages may promote tumor spread and help to explain the correlation between increased tumor-associated macrophage density and the augmented tumor vasculature recognized during experimental and human carcinogenesis. Moreover, macrophages take part in neovascularization by 'drilling' tunnels for new vasculature, producing tubular destruction of the matrix, distributing to form columns and capillary-like structures containing erythrocytes, ${ }^{8}$ localizing in microvessels embedded in bundles of fibrillar collagen ${ }^{9}$ and adhering to injured vessel walls, thus accelerating re-endothelization of the vascular barrier. ${ }^{10}$

In multiple myeloma (MM), bone marrow stromal cells increase the concentration of angiogenic factors and matrixdegrading enzymes in the bone marrow microenvironment by direct secretion, or by stimulation of myeloma cells or endothelial cells through paracrine interactions. ${ }^{11-13}$ Bone marrow stromal cells, osteoclasts, osteoblasts and endothelial cells secrete several factors, including vascular endothelial growth factor, fibroblast growth factor-2, tumor necrosis factor alpha, interleukin-6, B-cell activating factor, stromal cellderived factor $1 \alpha$ (also known as CXCL12) and various Notch family members, which are further upregulated by tumor cell adhesion to extracellular matrix proteins and/or bone marrow stromal cells. ${ }^{14}$ Bone marrow stromal cells and other accessory cells supporting MM cell survival in the bone microenvironment constitute potential therapeutic targets.
We have recently shown that when bone marrow macrophages from MM patients are exposed to vascular endothelial growth factor and fibroblast growth factor-2, which are major angiogenic cytokines secreted by plasma cells, ${ }^{15,16}$ and present in the bone marrow microenvironment at four- to five-fold higher levels than in peripheral blood, ${ }^{17}$ they transform into cells functionally and phenotypically similar to paired MM endothelial cells, and generate capillary-like networks mimicking those of MM endothelial cells. ${ }^{18}$ By contrast, macrophages from non-active MM, monoclonal gammopathies of undetermined significance and benign anemia patients display similar, albeit weaker features. Endothelial cell-like macrophages and apparently typical macrophages contribute sizeably to the formation of the neovessel wall in patients with active $M M$, whereas their vascular supply is minimal in non-active MM, and absent in MGUS patients and control patients. ${ }^{18}$ In patients with active MM, fluorescence-activated cell sorting (FACS) analyses on freshly isolated bone marrow mononuclear cells showed higher percentages of CD14/CD68 double-positive cells than in those with nonactive disease and MGUS. Furthermore, in active MM patients, bone marrow biopsies displayed macrophages with both endothelial cell-like (that is, CD68/FVIII-RA double positive) and apparently typical (that is, CD68 positive/FVIII-RA negative) features located in the microvessel wall and collaborating with $\mathrm{MM}$ endothelial cells to line the vessel lumen. Figures of this type were rare in non-active MM patients and absent in MGUS. Thus, macrophage involvement in the vasculogenic pathway proceeds in step with MM activity, and with progression of plasma cell tumors as well. ${ }^{18}$ Overall, these data suggest that in active MM, macrophages contribute to neovascularization through a vasculogenic pathway.

Chen et al. ${ }^{19}$ showed that monocytes induces vascular endothelial cell gene expression and develop tube-like structures when they are cultured with bone marrow from patients with MM that express an angiogenic factor, namely, pleiotrophin, effect specifically blocked with antibodies antipleiotrophin. Moreover, when co-injected with human MM cells into SCID mice, green fluorescent protein-marked human monocytes were found incorporated into tumor blood vessels and expressed human vascular endothelial cell protein markers and genes that were blocked by anti-pleiotrophin antibodies. These results suggest that vasculogenesis in human MM may develop from tumoral production of pleiotrophin, which induces the transdifferentiation of monocytes into vascular endothelial cells.

During embryo life, blood vessels first seem as the result of vasculogenesis, that is, the formation of capillaries from endothelial cells differentiating in situ from groups of mesodermal cells. The primitive heart and primitive vascular plexus form in this way. ${ }^{20}$ Remodeling of the primary vascular plexus into a more mature vascular system is thought to occur by angiogenesis, a term applied to the formation of capillaries from existing vessels, that is, capillaries and post-capillary venules, ${ }^{21}$ on the basis of endothelial sprouting or intussusceptive (nonsprouting) microvascular growth. ${ }^{21}$ In tumors, near angiogenesis, vasculogenesis contributes to the formation of the microvascular plexus that is important for local and systemic diffusion. ${ }^{22}$ 


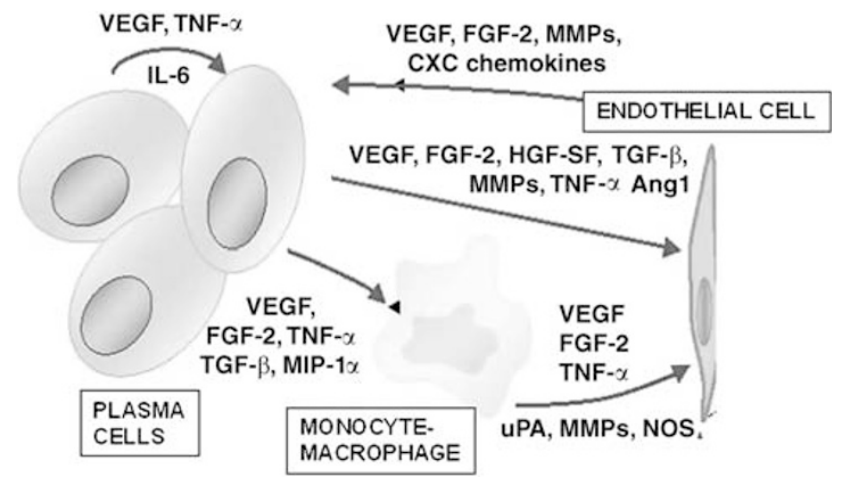

Figure 1 Interplay between plasma cells, monocytes-macrophages and endothelial cells in multiple myeloma. VEGF, vascular endothelial growth factor; MMPs, matrix metalloproteinases; TNF- $\alpha$, tumor necrosis factor alpha; TGF- $\beta$, transforming growth factor beta; IL-6, interleukin-6; FGF-2, fibroblast growth factor-2. Ang 1, angiopoietin 1; CXC, two $\mathrm{N}$-terminal cysteines of chemoxines separated by one amino acid (X); uPA, urokinase plasminogen activator; HCG-SF, hepatocyte growth factor-scatter factor; MIP- $1 \alpha$, macrophage inflammatory protein 1 alpha; NOS, nitric oxide synthase.

Experimental data reported here documented that in $\mathrm{MM}$, in association to angiogenesis, ${ }^{23}$ vasculogenesis also occurs and monocyte-macrophages participate actively in this process (Figure 1), together with hematopoietic stem and progenitor cells, as we have recently shown. ${ }^{24}$

The ability of tumor to support continued growth through non-angiogenesis-dependent pathways represents an important mechanism of tumor resistance to conventional anti-angiogenic treatment, and requires adjustment of these therapies to counter such new pathways.

\section{Conflict of interest}

The authors declare no conflict of interest.

\section{Acknowledgements}

This work was supported in part by grants from Associazione Italiana per la Ricerca sul Cancro (AIRC), Milan, Italy; the Ministry of Education, University and Research (MIUR, PRIN Projects 2007), Rome, Italy; the Ministry of Health, Progetto Oncologia 2006, Rome, Italy; Humanitas Mirasole S.p.A., Rome, Italy; Fondazione Cassa di Risparmio di Puglia, Bari, Italy.

\section{Ribatti ${ }^{1}$ and A Vacca ${ }^{2}$ \\ ${ }^{1}$ Department of Human Anatomy and Histology, University of Bari Medical School, Bari, Italy and \\ ${ }^{2}$ Department of Internal Medicine and Clinical Oncology, University of Bari Medical School, Bari, Italy E-mail: ribatti@anatomia.uniba.it}

\section{References}

1 Mantovani A, Bottazzi B, Colotta F, Sozzani S, Ruco L. The origin and function of tumor-associated macrophages. Immunol Today 1992; 13: 265-270.

2 Balkwill F, Mantovani A. Inflammation and cancer: back to Virchow. Lancet 2001; 357: 539-545.

3 Sunderkotter C, Goebeler M, Schiltze-Osthoff K, Bhardway R, Sorg C. Macrophages-derived angiogenesis factors. Pharmacol Ther 1991; 51: 195-216.
4 Lewis CE, Leek R, Harris A, Mc Gee JO. Cytokine regulation of angiogenesis in breast cancer: the role of tumor-associated macrophages. J Leukoc Biol 1995; 57: 747-751.

$5 \mathrm{Klimp} A \mathrm{H}$, Hollema H, Kempinga C, van der Zee AG, De Vries EG, Daemen T. Expression of cyclooxygenase-2 and inducible nitric oxide synthase in human ovarian tumors and tumor-associated macrophages. Cancer Res 2001; 61: 7305-7309.

6 Ribatti D, Nico B, Crivellato E, Vacca A. Macrophages and tumor angiogenesis. Leukemia 2007; 21: 2085-2089.

7 Zins K, Abraham D, Sioud M, Aharinejad S. Colon cancer cell-derived tumor necrosis factor-alpha mediated the tumor growth-promoting response in macrophages by up-regulating the colony-stimulating factor-1 pathway. Cancer Res 2007; 67: 1038-1045.

8 Moldovan NI, Goldschmidt-Clermont PJ, Parker-Thornburg J, Shapiro SD, Kolattukudy E. Contribution of monocytes/macrophages to compensatory neovascularization: the drilling of metalloelastase-positive tunnels in ischemic myocardium. Circ Res 2000; 87: 378-384.

9 Anghelina M, Krishnan P, Moldovan L, Moldovan NI. Monocytes/ macrophages cooperate with progenitor cells during neovascularization and tissue repair: conversion of cell columns into fibrovascular bundles. Am J Pathol 2006; 168: 529-541.

10 Fujiyama S, Amano K, Uehira K, Yoshida M, Nishiwaki Y, Nozawa Y et al. Bone marrow monocyte lineage cells adhere on injured endothelium in a monocyte chemoattractant protein-1-dependent manner and accelerate reendothelialization as endothelial progenitor cells. Circ Res 2003; 93: 980-989.

11 Rajkumar SV, Leong T, Roche PC, Fonseca R, Dispenzieri A, Lacy $\mathrm{MQ}$ et al. Prognostic value of bone marrow angiogenesis in multiple myeloma. Clin Cancer Res 2000; 6: 3111-3116.

12 Rajikumar SV, Mesa RA, Fonseca R, Schroeder G, Plavak MF, Dispenzieri $\mathrm{A}$ et al. Bone marrow angiogenesis in 400 patients with monoclonal gammopathy of undetermined significance, multiple myeloma, and primary amyloidosis. Clin Cancer Res 2002; 8: 2210-2216.

13 Ribatti D, Nico B, Vacca A. Importance of the bone marrow microenvironment in inducing the angiogenic response in multiple myeloma. Oncogene 2006; 25: 4257-4266.

14 Hideshima T, Mitsiades C, Tonon G, Richardson PG, Anderson $\mathrm{KC}$. Understanding multiple myeloma pathogenesis in the bone marrow to identify new therapeutic targets. Nat Rev Cancer 2007; 7: 585-598.

15 Bellamy WT, Richter L, Frutiger Y, Grogan TM. Expression of vascular endothelial growth factor and its receptors in hematological malignancies. Blood 1999; 59: 728-733.

16 Vacca A, Ribatti D, Presta M, Minischetti M, lurlaro M, Ria R et al. Bone marrow neovascularization, plasma cell angiogenic potential and matrix metalloproteinase- 2 secretion parallel progression of human multiple myeloma. Blood 1999; 93: 3064-3073.

17 Di Raimondo F, Azzaro MP, Palombo G, Bagnato S, Giustolisi G, Floridia $\mathrm{P}$ et al. Angiogenic factors in multiple myeloma: higher levels in bone marrow than in peripheral blood. Haematologica 2000; 85: 800-805.

18 Scavelli C, Nico B, Cirulli T, Ria R, Di Pietro G, Mangieri D et al. Vasculogenic mimicry by bone marrow macrophages in patient with multiple myeloma. Oncogene 2008; 27: 663-674.

19 Chen H, Campbell RA, Chang Y, Li M, Wang CS, Li J et al. Pleiotrophin produced by multiple myeloma induces transdifferentiation of monocytes into vascular endothelial cells: a novel mechanism of tumor-induced vasculogenesis. Blood; 2009; 113: 1992-2002.

20 Risau W, Flamme I. Vasculogenesis. Annu Rev Cell Dev Biol 1995; 11: 73-91.

21 Risau W. Mechanisms of angiogenesis. Nature 1997; 386: 671-674.

22 Ribatti D, Vacca A, Nico B, Roncali L, Dammacco F. Postnatal vasculogenesis. Mech Dev 2001; 100: 157-163.

23 Vacca A, Ribatti D. Bone marrow angiogenesis in multiple myeloma. Leukemia 2006; 20: 193-199.

24 Ria R, Piccoli C, Cirulli T, Falzetti F, Mangialardi G, Guidolin D et al. Endothelial differentiation of hematopoietic stem and progenitor cells from patients with multiple myeloma. Clin Cancer Res 2008; 14: 1678-1685. 